\title{
ON THE GELFAND-HILLE THEOREMS
}

\author{
JAROSLAV ZEMÁNEK \\ Institute of Mathematics, Polish Academy of Sciences \\ P.O. Box 137, 00-950 Warszawa, Poland \\ E-mail: zemanek@impan.impan.gov.pl
}

Let $T$ be a bounded linear operator on a complex Banach space $X$, with smallest possible spectrum, say, $\sigma(T)=\{1\}$. Thus, the resolvent $(T-\lambda I)^{-1}$ is an analytic function of $\lambda$ on $\mathbb{C} \backslash\{1\}$, vanishing at infinity, and the point 1 is either a pole or an essential singularity. More precisely, it is a pole of order $r$ if and only if $r$ is the least exponent such that $(T-I)^{r}=0$, because

$(T-\lambda I)^{-1}=-I(\lambda-1)^{-1}-(T-I)(\lambda-1)^{-2}-\ldots-(T-I)^{n}(\lambda-1)^{-(n+1)}-\ldots$

for $\lambda \neq 1$. This paper is devoted to characterizing the various situations, with emphasis on the case $r=1$. It relies on connections with complex analysis.

If $\operatorname{dim} X<\infty$, then $\sigma(T-I)=\{0\}$ implies that $T-I$ is nilpotent, hence there is a pole at 1 . The first result pertaining to the infinite-dimensional case was published by I. Gelfand [1941b]:

Theorem 1. Let $T \in B(X)$ be such that $\sigma(T)=\{1\}$. If $\sup _{n \in \mathbb{Z}}\left\|T^{n}\right\|<\infty$, then $T=I$.

The original proof was not so simple as the statement above, and it was not clear until 1950 whether all the assumptions (in particular, the boundedness of both positive and negative powers) are really needed for the conclusion. Also N. Dunford and E. Hille struggled with this problem around 1943, as is obvious from footnote ${ }^{14}$ ) in [N. Dunford 1943, p. 216]. Finally, G. E. Shilov [1950] gave an example showing that the boundedness of just the positive powers is not sufficient in Theorem 1.

1991 Mathematics Subject Classification: Primary 47A10, 47A35, 47D03; Secondary 30B30, $30 \mathrm{D} 15$.

The paper is in final form and no version of it will be published elsewhere. 
The following is a more transparent example. Let $V$ be the Volterra operator on the Hilbert space $L_{2}[0,1]$, defined by

$$
(V f)(t)=\int_{0}^{t} f(s) d s .
$$

It is well known that $\sigma(V)=\{0\}$, cf. [P. R. Halmos 1967, Problem 146]. Thus, the operator $T=(I+V)^{-1}$ has spectrum $\sigma(T)=\{1\}$. Moreover, it is not difficult to show that $\left\|T^{n}\right\|=1$ for $n \in \mathbb{N}$, cf. [P. R. Halmos 1967, Problem 150]. But $T \neq I$, because $V \neq 0$. Notice that the exponential formula [A. Pazy 1983, Theorem 1.8.3] yields $\left\|e^{t V}\right\|=1$ for $t<0$, so that the operator $e^{-V}$ provides yet another example; concerning this, see also [B. Aupetit 1991, Theorem 6.4.6], [G. Lumer and R. S. Phillips 1961, Theorem 2.3], and [R. Sine 1969]. (An interesting characterization of the Volterra operator was found by D. Przeworska-Rolewicz and S. Rolewicz [1987]. For other properties of $V$ see [V. S. Shul'man 1994] in this volume.) Nevertheless, Theorems 5 and 6 below, and also Theorems 7 and 8 for Riesz operators, show some other characterizations involving the positive powers of $T$ or $T-I$ only.

In connection with the latter example, let us recall that if $\left\|e^{i t S}\right\|=1$ for all $t \in \mathbb{R}$, where $S$ is a Hilbert space operator, then $S$ is selfadjoint. This originates from [I. Vidav 1956]. The following simple proof uses the Lie-Trotter formula [B. Aupetit 1991, Exercise III.15], [D. Petz 1994, Lemma 4] in this volume, [M. Reed and B. Simon 1972, Theorem VIII.29]. Indeed, it is enough to show that the selfadjoint operator $A=i\left(S-S^{*}\right)$ has zero spectrum. Since $\sigma(A) \subset \mathbb{R}$, the claim comes from

$$
\begin{aligned}
\left|\sigma\left(e^{t A}\right)\right| & \leq\left\|e^{i t\left(S-S^{*}\right)}\right\|=\left\|\lim _{n \rightarrow \infty}\left(e^{i t S / n} e^{-i t S^{*} / n}\right)^{n}\right\| \\
& \leq \limsup _{n \rightarrow \infty}\left(\left\|e^{i t S / n}\right\| \cdot\left\|e^{-i t S^{*} / n}\right\|\right)^{n}=1 .
\end{aligned}
$$

Notice that the weaker condition $\sup _{t \in \mathbb{R}}\left\|e^{i t P}\right\|<\infty$ is satisfied, for instance, by any $P=P^{2}$, not necessarily selfadjoint [B. Barnes 1989, p. 215]. This thread of development, closely related to the main topic of the present paper, can be traced in [B. Aupetit and D. Drissi 1994], [B. Aupetit and J. Zemánek 1990], [F. F. Bonsall and J. Duncan 1971; 1973], [R. S. Doran and V. A. Belfi 1986], [S. Kantorovitz 1965], [G. Lumer 1961; 1964], [I. Vidav 1982], and [B. Zalar 1993]. We shall see later on that the exponential function can also be used in proving Theorem 1.

It was E. Hille [1944] who pointed out that Theorem 1 and its generalization, Theorem 2 below, are consequences of an earlier result in complex analysis. To describe this, suppose that $F(\lambda)$ is an analytic function on $\mathbb{C} \backslash\{1\}$, vanishing at infinity, with Taylor series

$$
F(\lambda)=a_{0}+a_{1} \lambda+a_{2} \lambda^{2}+\ldots \text { for }|\lambda|<1,
$$


and

$$
F(\lambda)=b_{1} \lambda^{-1}+b_{2} \lambda^{-2}+\ldots \quad \text { for }|\lambda|>1 .
$$

Going back to L. Leau [1899], E. Le Roy [1900], S. Wigert [1900], and G. Faber [1903], consider the function

$$
G(z)=\frac{1}{2 \pi i} \int F(\lambda) e^{-(z+1) \log \lambda} d \lambda,
$$

where the integration is over a small circle around 1. This entire function has mild growth (zero exponential type of order one, cf. [L. Bieberbach 1927, Section VII.3]), which permits us to conclude that it is a polynomial of degree less than $r$ whenever the values at the integers satisfy $G(n)=o\left(n^{r}\right)$, or $G(n)=$ $O\left(n^{r-1}\right)$, as $|n| \rightarrow \infty$. The conclusion comes from the solutions by G. Szegö [1934] and L. Tschakaloff [1934] of a problem posed by G. Pólya [1931a]. This in turn implies that the original function $F(\lambda)$ has a pole of order at most $r$ at 1, cf. [P. Dienes 1931, p. 337] or [N. Obreschkoff 1934]. Further related literature includes [N. U. Arakelyan and V. A. Martirosyan 1991], [L. Bieberbach 1955], [R. P. Boas, Jr. 1954], [E. Hille 1962], [J. Korevaar 1948; 1949a; 1949b], [B. Ja. Levin 1964], [E. Lindelöf 1905], [A. I. Markushevich 1976], [R. E. A. C. Paley and N. Wiener 1934], [G. Pólya 1974], [A. Pringsheim 1932], [I. I. Privalov 1950], [S. L. Segal 1981], [M. H. Stone 1948], [G. Valiron 1925], and [D. V. Widder 1941].

The Pólya theorem [1931a] is a discrete version of the Bernstein inequality [1923], cf. [R. P. Boas, Jr. 1954, Theorem 11.1.2]. For a historical account of the latter see [N. I. Akhiezer 1951] and [P. R. Boas, Jr. 1969].

For applications to operator theory it is important to know that

$$
G(n)=a_{n} \text { and } \quad G(-n)=-b_{n}
$$

for $n \in \mathbb{N}$, cf. [N. Obreschkoff 1934] and [L. Bieberbach 1927, p. 289]. The particular function $F(\lambda)=(T-\lambda I)^{-1}$, with $\sigma(T)=\{1\}$, yields

$$
a_{n}=T^{-n-1} \quad \text { and } \quad b_{n}=-T^{n-1}
$$

for $n \in \mathbb{N}$. This together with the results mentioned before gives immediately the following theorem of E. Hille [1944], see also [M. H. Stone 1948] and [E. Hille and R. S. Phillips 1957, Theorem 4.10.1].

TheOREM 2. Let $T \in B(X)$ be such that $\sigma(T)=\{1\}$. Let $r$ be a positive integer. Then $(T-I)^{r}=0$ if and only if $\left\|T^{n}\right\|=o\left(n^{r}\right)$, or $\left\|T^{n}\right\|=O\left(n^{r-1}\right)$, as $|n| \rightarrow \infty$.

M. H. Stone [1948] obtained this by simplifying the proof of Pólya's theorem. G. E. Shilov [1950] pointed out that Theorem 2 can also be derived from [I. Gelfand 1941a]. Other proofs of Theorem 1 were discovered by A. Browder [1969] and G. Lumer [1971]. The latter gives an interesting estimate of $\|T-I\|$ by the behaviour of the spectrum on a path of finite length of elements joining $T$ to $I$ within a bounded commutative group, and is closely related to the result of 
A. Browder [1971], V. È. Katsnel'son [1970], and A. M. Sinclair [1971] (see also [F. F. Bonsall and M. J. Crabb 1970] and [B. Aupetit and D. Drissi 1994]) that the spectral radius is equal to the norm for a Hermitian operator on a Banach space in the sense of I. Vidav [1956].

Yet another proof and a generalization of Theorem 2 were obtained by T. Pytlik [1987]. The latter also follows from S. M. Shah's generalization [1946, Theorem 1] of Pólya's theorem [1931b]. Pólya's theorem [1931a] was also applied to Banach lattice homomorphisms by X.-D. Zhang [1992]. Elementary arguments in the context of Banach lattices can be found in [S. J. Bernau and C. B. Huijsmans 1990] and [H. H. Schaefer 1974, Proposition I.3.4].

The proof of Theorem 1 promised above in connection with the exponential function proceeds as follows. Since $\sigma(T)=\{1\}$, the holomorphic calculus [F. F. Bonsall and J. Duncan 1973, Theorems 7.4 and 7.6] or [E. Hille and R. S. Phillips 1957, Theorems 5.3.1 and 5.3.2] gives a $Q \in B(X)$ with $T=e^{Q}$ and $\sigma(Q)=\{0\}$. The entire function $e^{\lambda Q}$ is of the exponential type required in Pólya's theorem [1931a], by [F. F. Bonsall and J. Duncan 1973, Corollary 4.2], and its values at the integers, $T^{n}=e^{n Q}$, are bounded by Gelfand's assumption. Thus, the function is constant, which immediately yields $T=I$. Theorem 2 can be derived similarly by using Pólya's theorem as given in [R. P. Boas, Jr. 1954, Theorem 10.2.11] and [E. Hille and R. S. Phillips 1957, Theorem 3.13.8].

A significant step towards understanding Theorem 1 was made by J. Esterle [1983, Theorem 9.1] who proved, by an elegant argument based on the preceding exponential function and a Phragmén-Lindelöf theorem (a device also involved in Gelfand's proof), the following "half" counterpart to Theorem 1.

Theorem 3. Let $T \in B(X)$ be such that $\sigma(T)=\{1\}$. If $\sup _{n \in \mathbb{N}}\left\|T^{n}\right\|<\infty$, then $\left\|T^{n}-T^{n+1}\right\| \rightarrow 0$ as $n \rightarrow+\infty$.

Notice that Theorem 1 is an immediate consequence of Theorem 3.

In fact, knowing that

$$
T^{n}(T-I) \rightarrow 0 \quad \text { as } n \rightarrow+\infty, \text { and } \sup _{n \in \mathbb{N}}\left\|T^{-n}(T-I)\right\|<\infty,
$$

one sees that $(T-I)^{2}=0$. This implies that the sequence $\left\{T^{n}(T-I)\right\}$ is constant, because

$$
T^{n}(T-I)-T^{n+1}(T-I)=-T^{n}(T-I)^{2}=0 .
$$

Hence $T^{n}(T-I)=0$. Multiplication by $T^{-n}$ yields $T=I$. This argument suggests the following generalization of Theorems 1 and 2 .

Theorem 4. Let $T \in B(X)$ be invertible.

$1^{\circ}$ If $\left\|T^{n}-T^{n+1}\right\| \rightarrow 0$ as $n \rightarrow+\infty$, and $\sup _{n<0}\left\|T^{n}-T^{n+1}\right\|<\infty$, then $T=I$

$2^{\circ}$ If $\left\|T^{n}-T^{n+1}\right\|=O\left(n^{r-1}\right)$ as $|n| \rightarrow \infty$, for some positive integer $r$, then each isolated point of $\sigma(T)$ is a pole of order not exceeding $r+1$; in particular, if $\sigma(T)=\{1\}$, then $(T-I)^{r+1}=0$. 
Pr o of. Part $1^{\circ}$ sums up the preceding elementary argument. Part $2^{\circ}$ follows from [S. M. Shah 1946, Theorem 1] applied to the function $F(\lambda)=(\lambda-1)(T-$ $\lambda I)^{-1}$ whose Taylor coefficients are $a_{n}=T^{-n}-T^{-n-1}$ on $|\lambda|<1$, and $b_{n}=$ $T^{n-1}-T^{n}$ on $|\lambda|>1$, for $n \in \mathbb{N}$; the particular case comes directly from Pólya's theorem $[1931 \mathrm{~b}]$.

The matrix $T=\left(\begin{array}{ll}1 & 1 \\ 0 & 1\end{array}\right)$ illustrates the difference between Theorem 4 and the preceding results.

An apparently more general result than Theorem 3 (the same conclusion, and the same condition on the positive powers of $T$, but a weaker condition on $\sigma(T)$ allowing it to have points inside the unit disk, possibly accumulating at 1) was published by Y. Katznelson and L. Tzafriri [1986]; see also [C. J. K. Batty 1994b, Corollary 2.2] in this volume, for the precise formulation and some other related references. However, Vũ Quôc Phóng [1992] gave an elegant proof reducing the problem to the case where $\sigma(T)=\{1\}$, and applying Theorem 1 . Thus Theorem 3 is really the essential case. It is curious that [J. Esterle 1983] is not even referenced in [Y. Katznelson and L. Tzafriri 1986]. Another simple approach can be found in [G. R. Allan and T. J. Ransford 1989] and [P. Meyer-Nieberg 1991, Section 4.6].

The quantitative behaviour of the sequence $\left\{\left\|T^{n}-T^{n+1}\right\|\right\}$ as $n \rightarrow+\infty$ is studied in [O. Nevanlinna 1993, Chapter 4]. It does not mention the interesting result of J. Esterle [1983, Corollary 9.5], with the lower bound obtained by M. Berkani [1983, Corollaire 5.1.2]: If $\sigma(T)=\{1\}$ and $T \neq I$, then

$$
\liminf _{n \rightarrow+\infty} n\left\|T^{n}-T^{n+1}\right\| \geq 1 / 12 \text {. }
$$

Can this result be improved knowing that there is an essential singularity or a pole $\left({ }^{1}\right)$ of a given order at 1 ?

Next, the natural question arises whether it is possible to split Theorem 2 in a way similar to Theorem 3 , at least in the case when $\left\|T^{n}\right\|=o(n)$ as $n \rightarrow$ $+\infty$. Notice that the latter condition is necessary for $\left\|T^{n}-T^{n+1}\right\| \rightarrow 0$ as $n \rightarrow$ $+\infty$. The question of sufficiency was raised by G. R. Allan [1989, p. 7]. One can also ask whether the converse to the implication in Theorem 3 holds. However, A. Atzmon [in preparation] claims having a negative answer to both questions: for this purpose, translation operators on Banach spaces of special entire functions seem suitable; the highly interesting details await publication.

A negative answer to the second question, with $T=I-V$, can also be derived from the example in [T. Pytlik 1987, p. 292-293] by using the Fejér formula [H. Bateman, A. Erdélyi et al. 1953, p. 199], [G. Szegö 1959, Theorems 8.22.1 and 7.6.4], cf. also [G. Sansone 1959, p. 348] and [F. G. Tricomi 1955, p. 242].

The conclusion of Theorem 3 and the Fatou-Riesz theorem (see, for instance, [C. J. K. Batty 1994a, Theorem 1.6] in this volume, [P. Dienes 1931, p. 469], [E. Landau 1946, §18], [E. C. Titchmarsh 1939, Theorem 7.3.1], [G. Valiron 1954, $\S 20])$ guarantee the (weak) convergence of the exterior Taylor series $(|\lambda|>1)$ of

$\left.{ }^{1}\right)$ Krzysztof Bolibok observed that in this case the limit is $+\infty$ (March 9, 1994). 
the function $(\lambda-1)(T-\lambda I)^{-1}$ at the points of the unit circle different from 1 , where the function has analytic extension. These boundary values can then be extended, by the Abel theorem (see, for instance, [P. Dienes 1931, p. 102], [K. Knopp 1947, p. 179], [R. Remmert 1991, p. 120]), to a new power series on the open unit disk. If one knows that the interior Taylor series $(|\lambda|<1)$ of $(\lambda-1)(T-\lambda I)^{-1}$ also (weakly) converges at the same points of the unit circle, then the radial limits of the two Taylor series coincide at these points and, finally, the Lusin-Privalov theorem (see, for instance, [E. F. Collingwood and A. J. Lohwater 1966, Corollary 8.3], [K. Noshiro 1960, §III.3], [I. I. Privalov 1950, pp. 319-320]) implies that the two series coincide, which gives the conclusion of Theorem 1 . It is this difficulty that had to be overcome by the other analytic tools involved in Theorems 2 and 3 .

Theorem 2 was used by J. Wermer [1952] in proving that an invertible operator $T$ on a Banach space has a non-trivial invariant subspace if $\left\|T^{n}\right\|=O\left(n^{r}\right)$ as $|n| \rightarrow \infty$, for some $r=0,1,2, \ldots$; it seems interesting to note that (a more general form of) this condition as well as (a particular case of) the spectral radius formula appear already in [A. Beurling 1938]. H. Radjavi and P. Rosenthal [1973, Theorem 6.4] give another proof based on a resolvent growth condition. Local versions of Wermer's theorem can be found in [A. Atzmon 1984] and [B. Beauzamy 1988]. For operators with general spectra, the aim of these results is to conclude that, under certain growth conditions on the iterates of $T$, either $T=I$ or $T$ has a non-trivial hyperinvariant subspace. See also [S. Grabiner 1979, Theorem 4.1]. The quantitative behaviour of the powers has also influence on the structure of invariant subspaces of an operator, cf. [A. Atzmon 1993]. In general, this development has led to a better understanding of Theorem 2 .

Recall that the condition $\sigma(T)=\{1\}$ is equivalently expressed by requiring that $\left\|(T-I)^{n}\right\|^{1 / n} \rightarrow 0$ as $n \rightarrow+\infty$. It was observed by A. Atzmon [1980, Corollary 7] that a stronger requirement on the rate of convergence in the preceding spectral radius formula (anyway necessary for $T-I$ to be nilpotent) makes it possible to drop the assumption on the negative powers of $T$; the analytic device underlying this is again [G. Pólya 1931a].

TheOREM 5. Let $T \in B(X)$ be such that $n\left\|(T-I)^{n}\right\|^{1 / n} \rightarrow 0$ as $n \rightarrow+\infty$. If $\left\|T^{n}\right\|=O\left(n^{r-1}\right)$ as $n \rightarrow+\infty$, for some positive integer $r$, then $(T-I)^{r}=0$.

Characterizations of nilpotent elements in terms of conditions on the growth of the resolvent can be found in [I. Gelfand 1941a], [O. Nevanlinna 1993, Theorem 5.4.1], [J. G. Stampfli 1967], and [J. G. Stampfli and J. P. Williams 1968, Theorem 7]. The behaviour of the spectrum near such elements was studied by B. Aupetit and J. Zemánek [1981; 1983].

Recall that an operator $T$ is said to have finite descent, equal to $r$, if $r$ is the smallest non-negative integer such that $R\left(T^{r}\right)=R\left(T^{r+1}\right)$, where $R(T)=T X$ is the range of $T$. The ascent is defined similarly with respect to the behaviour of the null space $N(T)=T^{-1}(0)$ of the iterates of $T$. See [A. E. Taylor and D. C. Lay 1980, p. 290], and remember that for a general operator such finite 
numbers may not exist. However, if they both exist, then they are equal [ibid., Theorem V.6.2], and 0 is a pole of the resolvent of order $r$; in fact, this is a geometric characterization of a pole [ibid., p. 330]:

$$
X=N\left(T^{r}\right) \oplus R\left(T^{r}\right),
$$

where $R\left(T^{r}\right)$ is automatically closed by [ibid., Theorem IV.5.10].

Gelfand's case $r=1$ admits more characterizations. They are listed in the next theorem, where the notation

$$
M_{n}(T)=\frac{I+T+\ldots+T^{n-1}}{n}, \quad n \in \mathbb{N},
$$

and $M_{-n}(T)=M_{n}\left(T^{-1}\right)$, is used.

TheOREM 6. Let $T \in B(X)$. The following conditions are equivalent:

$1^{\mathrm{o}} \sigma(T)=\{1\}$, and $\sup _{n \in \mathbb{Z}}\left\|M_{n}(T)\right\|<\infty$;

$2^{\circ} \sigma(T)=\{1\}$, and the sequence $\left\{M_{n}(T) ; n \in \mathbb{N}\right\}$ is convergent in $B(X)$;

$3^{\circ} \sigma(T)=\{1\}$, and the set $\left\{T^{n} ; n \in \mathbb{N}\right\}^{-}$is compact in $B(X)$;

$4^{\mathrm{o}} \sigma(T)=\{1\}$, and $\lim \sup _{n \rightarrow+\infty}\left\|T^{n}-T^{n+1}\right\|^{1 / n}<1$;

$5^{\mathrm{o}} \sigma(T)=\{1\}$, and $\liminf _{n \rightarrow+\infty}\left\|T^{n}-T^{n+1}\right\|^{1 / n}<1$;

$6^{\circ} \sigma(T)=\{1\},\left\|T^{n}\right\|=o(n)$ as $n \rightarrow+\infty$, and $R\left((T-I)^{m}\right)$ is closed for some $m=1,2, \ldots$;

$7^{\circ} \sigma(T)=\{1\},\left\|T^{n} x\right\|=o(n)$ as $n \rightarrow+\infty$, for every $x \in X$, and $R\left((T-I)^{m}\right)$ is closed for some $m=2,3, \ldots$;

$8^{\circ} \sigma(T)=\{1\},\left\|T^{n} x\right\|=o(n)$ as $n \rightarrow+\infty$, for every $x \in X$, and $T-I$ has finite descent;

$9^{\circ}\left\|T^{n}\right\|=o(n)$ as $n \rightarrow+\infty$, and $\liminf _{n \rightarrow+\infty}\left\|I-M_{n}(T)\right\|<1$;

$10^{\circ} \sigma(T)=\{1\}$, and $\left\|(T-\lambda I)^{-1}\right\| \leq \mathrm{const} \cdot|\lambda-1|^{-1}$ for $\lambda$ in a deleted neighbourhood of 1 ;

$11^{\circ} \sigma(T)=\{1\}$, and $\left\|(T-\lambda I)^{-1}\right\| \leq \mathrm{const} \cdot|| \lambda|-1|^{-1}$ for $|\lambda| \neq 1$;

$12^{\circ} T=I$.

Proof and comments. It is enough to show that each condition implies $12^{\circ}$. The implication $1^{\circ} \Rightarrow 12^{\circ}$ is a simple application of the formula

$$
\frac{I-T^{n}}{n}=(I-T) M_{n}(T)
$$

and the case $r=2$ of Theorem 2, see [M. Mbekhta et J. Zemánek 1993, Théorème 2].

The implication $2^{\circ} \Rightarrow 12^{\circ}$ can also be derived from the preceding formula; it is a particular case of [N. Dunford 1943, Theorem 3.16].

Condition $3^{\circ}$ implies that 1 is a simple pole of the resolvent of $T$ (hence $T=I$ ), by the theorem of M. A. Kaashoek and T. T. West [1968, Theorem 3; 1974, Theorem I.2.3]; see also [M. A. Kaashoek 1969], [J. J. Koliha 1974a], [R. A. Hirschfeld 
1968], [L. J. Wallen 1967], and [A. Święch 1990]. Notice that the Kaashoek-West theorem has an earlier analogy in complex analysis [G. Pólya und G. Szegö 1964, Aufgabe III.241].

The implication $4^{\circ} \Rightarrow 12^{\circ}$ is a consequence of the corresponding result in complex analysis [A. Pringsheim 1929, p. 115; 1932, p. 917]; see also [N. U. Arakelyan and V. A. Martirosyan 1991, p. 57]. The implication $5^{\circ} \Rightarrow 12^{\circ}$ follows from the already mentioned result of J. Esterle [1983, Corollary 9.5].

The implication $6^{\circ} \Rightarrow 12^{\circ}$ can be found in [M. Mbekhta et J. Zemánek 1993, Corollaire 2].

The condition $\left\|T^{n} x\right\|=o(n)$ as $n \rightarrow+\infty$, for every $x \in X$, implies that $T-I$ has ascent at most 1 , cf. [ibid., Lemme]. Thus $8^{\circ}$ implies $12^{\circ}$ by [A. E. Taylor and D. C. Lay 1980, Theorem V.6.2 and p. 330], while $7^{\circ}$ implies $12^{\circ}$ by [D. C. Lay 1970, Theorem 2.7] or [S. Grabiner 1974, Theorem 5.4]. It is not clear whether $m=1$ can be allowed in condition $7^{\circ}$. More general forms of $8^{\circ} \Rightarrow 12^{\circ}$ are [S. Grabiner 1971, Theorem 2; 1974, Theorem 5.2; 1982, Corollary 4.9], [D. C. Lay 1970, Theorem 2.6], and [A. E. Taylor and D. C. Lay 1980, p. 332].

An elementary proof of the implication $9^{\circ} \Rightarrow 12^{\circ}$ can be found in [W. Wils 1969]; it is remarkable that this result has no spectral assumption.

Condition $10^{\circ}$ implies $12^{\circ}$ by the Laurent series development at 1 . It is included here because it can be split in two symmetric parts: condition $10^{\circ}$ restricted to $|\lambda|>1$ only yields the Hille condition $\left\|T^{n}\right\|=o(n)$ as $n \rightarrow+\infty$, by [R. K. Ritt 1953], and similarly for $|\lambda|<1$ it gives the analogous conclusion as $n \rightarrow-\infty$. Thus the result is also a consequence of Theorem 2. It would be interesting to know whether, conversely, the (one-sided) Hille condition implies the (one-sided) Ritt resolvent estimate. If not, is it then possible that the Ritt condition implies a stronger conclusion like $\left\|T^{n}-T^{n+1}\right\| \rightarrow 0$ as $n \rightarrow+\infty\left({ }^{2}\right)$, or conversely? Perhaps [E. C. Titchmarsh 1939, Example 7.8.16] and [N. K. Nikol'skiü 1977] could be of use here? A finite-dimensional version of the latter can be found in [L. Collatz $1963, \S 19.8]$.

Finally, condition $11^{\circ}$ implies that $\left\|T^{n}\right\|=O(n)$ as $|n| \rightarrow \infty$, by [I. Colojoară and C. Foias 1968, Proposition 5.1.6], hence $T=I$ by Theorem 2 and the Laurent series development at 1 . Alternatively, one can also show that $11^{\circ}$ implies $10^{\circ}$. The proof is complete.

It would be interesting to find analogous characterizations of a general pole

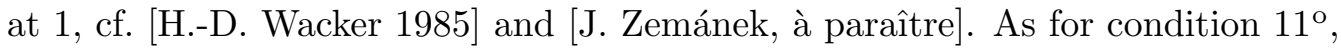
this is possible by [I. Colojoară and C. Foiaş, Proposition 5.1.6] and Theorem 2. Apart from the characterizations of poles and nilpotent elements already mentioned, a recent result in this direction is [C. Schmoeger 1993, Theorem 5]. Also L. Burlando [1994] follows this way.

Is there a link between Theorem 5 and conditions $4^{\circ}, 5^{\circ}$, or $9^{\circ}$ of Theorem 6 ?

$\left({ }^{2}\right)$ Yes, Olavi Nevanlinna verified this on March 9, 1994. 
Theorem 6 also suggests a number of other problems: If the spectral restriction $\sigma(T)=\{1\}$ is relaxed or dropped, what are the relationships between the remaining parts of the corresponding conditions? For instance, S. Grabiner (private communication) has constructed an operator with descent one and non-closed range. Interesting examples of relationships between various conditions involving the powers of operators and the resolvent can be found e.g. in [A. Atzmon 1983; 1993], [B. Beauzamy 1987; 1988], [A. Bernard 1971], [A. Brunel et R. Émilion 1984], [A. L. Bukhgel̆m 1988], [Y. Derriennic and M. Lin 1973], [W. F. Donoghue, Jr. 1963], [R. Emilion 1985], [C. Fernandez-Pujol 1988], [A. G. Gibson 1972], [I. Gohberg, S. Goldberg and M. A. Kaashoek 1990, p. 166], [I. C. Gohberg and M. G. Krĕ̌n 1969, p. 244], [L. K. Jones and M. Lin 1980], [J. J. Koliha 1974b], [G. K. Leaf 1963], [E. R. Lorch 1941], [C. Lubich and O. Nevanlinna 1991], [C. A. McCarthy 1971], [C. A. McCarthy and J. Schwartz 1965], [A. Mokhtari 1988], [V. Müller 1994], [O. Nevanlinna 1993], [T. Nieminen 1962], [H. C. Rönnefarth 1993], [A. L. Shields 1978], [B. M. Solomyak 1982; 1983], [J. C. Strikwerda 1989], [J. A. Van Casteren 1985], [F. Wolf 1957].

On the other hand, it would be interesting to know what happens to some of these results if a spectral restriction, like $\sigma(T)=\{1\}$, is imposed. Sometimes the spectral condition itself may imply $T=I$, cf. [W. Arendt 1983, Corollary 3.6].

B. Sz.-Nagy [1947] proved that a Hilbert space operator $T$ with $\sup _{n \in \mathbb{Z}}\left\|T^{n}\right\|<$ $\infty$ is similar to a unitary operator. What is the class of operators satisfying the weaker condition $\sup _{n \in \mathbb{Z}}\left\|M_{n}(T)\right\|<\infty$ ? For the Riesz operators, see assertion $3^{\circ}$ in Theorem 8 below. For general Hilbert space operators, related results can be found in [J. A. Van Casteren 1985, Theorem 7.10].

For special operators the situation simplifies. For instance, the following comes from [M. Mbekhta et J. Zemánek 1993, Théorème 3] and [J. I. Nieto 1982, Corollaire 1], correcting and completing [B. Beauzamy 1988, Exercise I.1].

TheOREM 7. Let $T \in B(X)$ be a Riesz operator. The following conditions are equivalent:

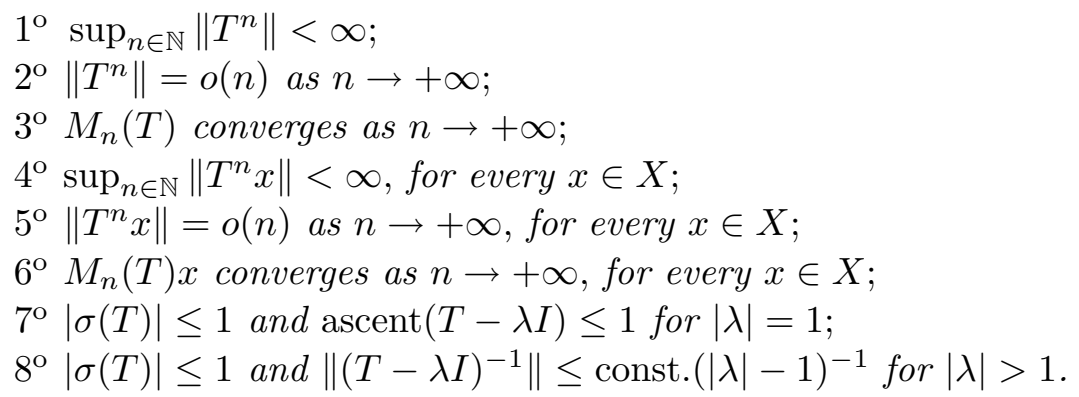

Notice that, in contrast to Theorems 1 and 2, one-sided conditions are sufficient in Theorem 7; this is also explained by an interesting inequality between $\left\|T^{-1}\right\|$ and $\|T\|$ in the finite-dimensional case, cf. [J. W. Daniel and T. W. Palmer 1969], [V. Pták 1976], and [N. J. Young 1978, Theorem 4]. 
Of course, $1^{\circ}$ and $4^{\circ}$ (of Theorem 7 ) are equivalent for any operator, by the Banach-Steinhaus theorem. What are the relations between the other conditions in general? A characterization of property $6^{\circ}$ can be found in [R. Sine 1970]; see also MR40\#5825, [S.-Y. Shaw 1980], and [R. Sato 1979; 1981]. The qualitative behaviour of orbits $\left\{T^{n} x\right\}$ was studied by S. Rolewicz [1969], and the subsequent development is traced by B. Beauzamy [1988]; see also [L. Kérchy 1994] and [V. Müller 1994] in this volume.

The Jordan theorem gives a spectral characterization of the following more general behaviour. Interesting related results and questions can be found in $[\mathrm{H}$. C. Rönnefarth 1993]. See also [J. C. Strikwerda and B. A. Wade 1991].

TheOREM 8. Let $T \in B(X)$ be a Riesz operator with $|\sigma(T)| \leq 1$.

$1^{\circ}\left\|T^{n}\right\|=O(n)$ as $n \rightarrow+\infty$ if and only if $\operatorname{ascent}(T-\lambda I) \leq 2$ for $|\lambda|=1$;

$2^{\circ} T^{n} / n$ converges as $n \rightarrow+\infty$ if and only if

$$
\begin{aligned}
& \operatorname{ascent}(T-I) \leq 2 \quad \text { and } \operatorname{ascent}(T-\lambda I) \leq 1 \quad \text { for }|\lambda|=1, \lambda \neq 1 ; \\
& 3^{\circ} \sup _{n \in \mathbb{N}}\left\|M_{n}(T)\right\|<\infty \text { if and only if } \\
& \quad \operatorname{ascent}(T-I) \leq 1 \text { and } \operatorname{ascent}(T-\lambda I) \leq 2 \quad \text { for }|\lambda|=1, \lambda \neq 1 .
\end{aligned}
$$

Is it possible to obtain similar characterizations of poles, on the unit circle, of order not exceeding a given number? Is it possible to replace the assumption $\sup _{n \in \mathbb{N}}\left\|T^{n}\right\|<\infty$ in Theorem 3 by $\sup _{n \in \mathbb{N}}\left\|M_{n}(T)\right\|<\infty$ ?

Is there a local version of Theorem 3? In this direction, see [W. Arendt and C. J. K. Batty 1988, Theorem 5.1], [C. J. K. Batty 1994b, Theorem 2.5] in this volume, and [Yu. I. Lyubich and Vũ Quôc Phóng 1988].

There are local versions of the Gelfand-Hille theorems.

Theorem 9. Let $T \in B(X)$ be such that $\sigma(T)=\{1\}$. Let $p$ and $q$ be positive integers, and let $x \in X$. Suppose that $\left\|T^{n} x\right\|=o\left(n^{p}\right)$ as $n \rightarrow+\infty$, and $\left\|T^{n} x\right\|=$ $o\left(n^{q}\right)$ as $n \rightarrow-\infty$. Then $(T-I)^{r} x=0$, where $r=\max (p, q)$. However, if $\min (p, q)=1$, then actually $T x=x$.

Proof. The first assertion follows from Pólya's theorem [1931b] applied to the function $F(\lambda)=(T-\lambda I)^{-1} x$.

For the second, suppose that $(T-I)^{r} x=0$ for some $r \geq 2$, and that, for instance, $\left\|T^{n} x\right\|=o(n)$ as $n \rightarrow+\infty$. Let $y=(T-I)^{r-1} x$. Then $(T-I) y=0$, hence $M_{n}(T) y=y$. On the other hand,

$M_{n}(T) y=(T-I)^{r-2} M_{n}(T)(T-I) x=(T-I)^{r-2} \frac{T^{n}-I}{n} x \rightarrow 0 \quad$ as $n \rightarrow+\infty$, hence $y=0$. By induction, $(T-I) x=0$.

The first assertion was obtained in [B. Aupetit and D. Drissi 1994] by a different method based on a theorem of B. Ja. Levin [1964]. In view of the second assertion, it would be interesting to know whether a similar improvement of the first assertion is possible. Global features of this phenomenon can also be observed 
in [J. Esterle 1994], [V. I. Istrăţescu 1978, Theorem 6.3.1], [Vũ Quôc Phóng 1993, Lemma 4], and [M. Zarrabi 1993, Corollaire 3.2; to appear]. Thus, there remains the feeling that the role of the two one-sided conditions in the Gelfand-Hille theorems is not quite symmetric.

Let us conclude with a problem arising in [M. Mbekhta et J. Zemánek 1993]. In conditions $6^{\circ}$ and $7^{\circ}$ of Theorem 6 it is important to know when (a power of) a quasinilpotent operator has closed range. In this context, the following instructive example was suggested by V. Müller and W. R. Wogen.

Let $Q$ be a quasinilpotent operator which is not nilpotent. Consider the operator

$$
S=\left(\begin{array}{cc}
Q & I \\
0 & 0
\end{array}\right) \quad \text { on } X \oplus X .
$$

Then $R(S)=X$ is closed, and $S$ is quasinilpotent but not nilpotent. Next, L. Burlando [1994] and, independently, W. R. Wogen claimed to have constructed a quasinilpotent operator which is not nilpotent and all of whose (positive) powers have closed range. Concerning this situation, see also [B. Johnson 1971, Lemma].

What can be said about the intersection of the ranges of the powers of a quasinilpotent operator? When is it non-zero? When is the range of a quasinilpotent operator dense in $X$ ? Can the answers to these questions be given in terms of the behaviour of the powers or the resolvent?

Acknowledgements. I am grateful to E. Abakumov, A. Atzmon, A. Bogomol'naya, K. Bolibok, L. Burlando, Ş. Frunză, T. A. Gillespie, S. Grabiner, V. Lomonosov, E. Makai, V. Matsaev, M. Mbekhta, V. Müller, O. Nevanlinna, N. K. Nikol'skiı̌, J. Siciak, B. Sz.-Nagy, A. Tonge, I. Vidav, and W. R. Wogen for valuable discussions on various questions of this paper.

\section{References}

N. I. Akhiezer [1951], The work of academician S. N. Bernšter̆n on the constructive theory of functions (on the occasion of his seventieth birthday), Uspekhi Mat. Nauk 6, no. 1, 3-67 (in Russian).

G. R. Allan [1989], Power-bounded elements in a Banach algebra and a theorem of Gelfand, in: Conference on Automatic Continuity and Banach Algebras (Canberra, January 1989), R. J. Loy (ed.), Proc. Centre Math. Anal. Austral. Nat. Univ. 21, 1-12.

G. R. Allan and T. J. Ransford [1989], Power-dominated elements in a Banach algebra, Studia Math. 94, 63-79.

N. U. Arakelyan and V. A. Martirosyan [1991], Power Series: Analytic Extension and Localization of Singularities, University of Erevan, Erevan (in Russian).

W. Arendt [1983], Spectral properties of Lamperti operators, Indiana Univ. Math. J. 32, 199-215.

W. Arendt and C. J. K. Batty [1988], Tauberian theorems and stability of one-parameter semigroups, Trans. Amer. Math. Soc. 306, 837-852.

A. Atzmon [1980], Operators which are annihilated by analytic functions and invariant subspaces, Acta Math. 144, 27-63. 
A. Atzmon [1983], Operators with resolvent of bounded characteristic, Integral Equations Operator Theory $6,779-803$.

A. Atzmon [1984], On the existence of hyperinvariant subspaces, J. Operator Theory 11, 3-40.

A. Atzmon [1993], Unicellular and non-unicellular dissipative operators, Acta Sci. Math. (Szeged) 57, 45-54.

A. Atzmon [in preparation], On the asymptotic growth of the sequence $\left\{\left\|T^{n+1}-T^{n}\right\|\right\}_{n=1}^{\infty}$ for some operators $T$ with $\sigma(T)=\{1\}$.

B. Aupetit [1991], A Primer on Spectral Theory, Springer, New York.

B. A upetit and D. Drissi [1994], Some spectral inequalities involving generalized scalar operators, Studia Math. 109, 51-66.

B. A upetit and J. Zemánek [1981], Local behaviour of the spectral radius in Banach algebras, J. London Math. Soc. 23, 171-178.

B. A upetit and J. Zemánek [1983], Local behavior of the spectrum near algebraic elements, Linear Algebra Appl. 52/53, 39-44.

B. Aupetit and J. Zemánek [1990], A characterization of normal matrices by their exponentials, ibid. 132, 119-121; 180 (1993), 1-2.

B. A. Barnes [1989], Operators which satisfy polynomial growth conditions, Pacific J. Math. 138, 209-219.

H. Bateman, A. Erdélyi et al. [1953], Higher Transcendental Functions II, McGraw-Hill, New York.

C. J. K. Batty [1994a], Some Tauberian theorems related to operator theory, this volume, 21-34

C. J. K. Batty [1994b], Asymptotic behaviour of semigroups of operators, this volume, 35-52.

B. Beauzamy [1987], Orbites tendant vers l'infini, C. R. Acad. Sci. Paris Sér. I Math. 305, $123-126$.

B. Beauzamy [1988], Introduction to Operator Theory and Invariant Subspaces, North-Holland, Amsterdam.

M. Berkani [1983], Inégalités et Propriétés Spectrales dans les Algèbres de Banach, Thèse, Université de Bordeaux I, Bordeaux.

A. Bernard [1971], Algèbres quotients d'algèbres uniformes, C. R. Acad. Sci. Paris Sér. A-B 272, A1101-A1104.

S. J. Bernau and C. B. Huijsmans [1990], On the positivity of the unit element in a normed lattice ordered algebra, Studia Math. 97, 143-149.

S. Bernstein [1923], Sur une propriété des fonctions entières, C. R. Acad. Sci. Paris 176, 1603-1605.

A. Beurling [1938], Sur les intégrales de Fourier absolument convergentes et leur application $\grave{a}$ une transformation fonctionnelle, in: Ninth Scandinavian Math. Congress, Helsingfors, 345-366. Also in: Collected Works of Arne Beurling, Vol. 2, Harmonic Analysis, L. Carleson, P. Malliavin, J. Neuberger, and J. Wermer (eds.), Birkhäuser, Boston, 1989, 39-60.

L. Bieberbach [1927], Lehrbuch der Funktionentheorie II, Teubner, Berlin.

L. Bieberbach [1955], Analytische Fortsetzung, Springer, Berlin.

R. P. Boas, Jr. [1954], Entire Functions, Academic Press, New York.

R. P. Boas, Jr. [1969], Inequalities for the derivatives of polynomials, Math. Mag. 42, 165-174.

F. F. Bonsall and M. J. Crabb [1970], The spectral radius of a Hermitian element of a Banach algebra, Bull. London Math. Soc. 2, 178-180.

F. F. Bonsall and J. Duncan [1971], Numerical Ranges of Operators on Normed Spaces and of Elements of Normed Algebras, Cambridge University Press, Cambridge.

F. F. Bonsall and J. Duncan [1973], Complete Normed Algebras, Springer, Berlin.

A. Browder [1969], States, numerical ranges, etc., Proc. Brown Univ. Informal Analysis Seminar, Providence.

A. Browder [1971], On Bernstein's inequality and the norm of Hermitian operators, Amer. Math. Monthly 78, 871-873. 
A. Brunel et R. Émilion [1984], Sur les opérateurs positifs à moyennes bornées, C. R. Acad. Sci. Paris Sér. I Math. 298, 103-106.

A. L. Bukhgeı̆m [1988], Introduction to the Theory of Inverse Problems, Nauka, Novosibirsk (in Russian).

L. Burlando [1994], Characterizations of nilpotent operators, letter.

L. Collatz [1963], Eigenwertaufgaben mit technischen Anwendungen, Geest \& Portig, Leipzig.

E. F. Collingwood and A. J. Lohwater [1966], The Theory of Cluster Sets, Cambridge University Press, Cambridge.

I. Colojoară and C. Foiaş [1968], Theory of Generalized Spectral Operators, Gordon and Breach, New York.

J. W. Daniel and T. W. Palmer [1969], On $\sigma(T),\|T\|$, and $\left\|T^{-1}\right\|$, Linear Algebra Appl. 2, 381-386.

Y. Derriennic and M. Lin [1973], On invariant measures and ergodic theorems for positive operators, J. Funct. Anal. 13, 252-267.

P. Dienes [1931], The Taylor Series, Oxford University Press, Oxford.

W. F. Donoghue, Jr. [1963], On a problem of Nieminen, Inst. Hautes Études Sci. Publ. Math. 16, 127-129.

R. S. Doran and V. A. Belfi [1986], Characterizations of $C^{*}$-Algebras, Marcel Dekker, New York.

N. Dunford [1943], Spectral theory. I. Convergence to projections, Trans. Amer. Math. Soc. 54, 185-217.

R. Emilion [1985], Mean-bounded operators and mean ergodic theorems, J. Funct. Anal. 61, $1-14$.

J. Esterle [1983], Quasimultipliers, representations of $H^{\infty}$, and the closed ideal problem for commutative Banach algebras, in: Radical Banach Algebras and Automatic Continuity (Long Beach, Calif., 1981), J. M. Bachar, W. G. Bade, P. C. Curtis Jr., H. G. Dales, and M. P. Thomas (eds.), Lecture Notes in Math. 975, Springer, 66-162.

J. Esterle [1994], Uniqueness, strong forms of uniqueness and negative powers of contractions, this volume, $127-145$

G. Faber [1903], Über die Fortsetzbarkeit gewisser Taylorscher Reihen, Math. Ann. 57, 369388.

C. Fernandez-Pujol [1988], Séries convergentes d'opérateurs dans un espace de Banach, C. R. Acad. Sci. Paris Sér. I Math. 306, 331-334.

I. Gelfand [1941a], Ideale und primäre Ideale in normierten Ringen, Mat. Sb. 9, 41-48.

I. Gelfand [1941b], Zur Theorie der Charactere der Abelschen topologischen Gruppen, ibid. 9, $49-50$.

A. G. Gibson [1972], A discrete Hille-Yosida-Phillips theorem, J. Math. Anal. Appl. 39, 761-770.

I. Gohberg, S. Goldberg and M. A. Kaashoek [1990], Classes of Linear Operators I, Birkhäuser, Basel.

I. C. Gohberg and M. G. Kre ̌n [1969], Introduction to the Theory of Linear Nonselfadjoint Operators, Amer. Math. Soc., Providence.

S. Grabiner [1971], Ranges of quasi-nilpotent operators, Illinois J. Math. 15, 150-152.

S. Grabiner [1974], Ranges of products of operators, Canad. J. Math. 26, 1430-1441.

S. Grabiner [1979], Operator ranges and invariant subspaces, Indiana Univ. Math. J. 28, $845-857$.

S. Grabiner [1982], Uniform ascent and descent of bounded operators, J. Math. Soc. Japan 34, 317-337.

P. R. Halmos [1967], A Hilbert Space Problem Book, Von Nostrand, Princeton.

E. Hille [1944], On the theory of characters of groups and semi-groups in normed vector rings, Proc. Nat. Acad. Sci. U.S.A. 30, 58-60. 
E. Hille [1962], Analytic Function Theory II, Ginn, Boston.

E. Hille and R. S. Phillips [1957], Functional Analysis and Semi-Groups, Amer. Math. Soc., Providence.

R. A. Hirschfeld [1968], On semi-groups in Banach algebras close to the identity, Proc. Japan Acad. 44, 755 .

V. I. Istrățescu [1978], Topics in Linear Operator Theory, Academia Nazionale dei Lincei, Roma.

B. Johnson [1971], Continuity of operators commuting with quasi-nilpotent operators, Indiana Univ. Math. J. 20, 913-915.

L. K. Jones and M. Lin [1980], Unimodular eigenvalues and weak mixing, J. Funct. Anal. 35, $42-48$.

M. A. Kaashoek [1969], Locally compact semi-algebras and spectral theory, Nieuw Arch. Wisk. $17,8-16$

M. A. Kaashoek and T. T. West [1968], Locally compact monothetic semi-algebras, Proc. London Math. Soc. 18, 428-438.

M. A. Kaashoek and T. T. West [1974], Locally Compact Semi-Algebras with Applications to Spectral Theory of Positive Operators, North-Holland, Amsterdam.

S. Kantorovitz [1965], Classification of operators by means of their operational calculus, Trans. Amer. Math. Soc. 115, 194-224.

V. È. Katsnel'son [1970], Conservative operator has norm equal to its spectral radius, Mat. Issled. 5, no. 3, 186-189 (in Russian).

Y. Katznelson and L. Tzafriri [1986], On power bounded operators, J. Funct. Anal. 68, 313-328.

L. Kérchy [1994], Unitary asymptotes of Hilbert space operators, this volume, 191-201.

K. Knopp [1947], Theorie und Anwendung der unendlichen Reihen, Springer, Berlin.

J. J. Koliha [1974a], Some convergence theorems in Banach algebras, Pacific J. Math. 52, 467-473.

J. J. Koliha [1974b], Power convergence and pseudoinverses of operators in Banach spaces, J. Math. Anal. Appl. 48, 446-469.

J. Korevaar [1948], Entire functions of exponential type, Math. Centrum Amsterdam, Rapport ZW 1948-011, 10 pp. (in Dutch).

J. Korevaar [1949a], Functions of exponential type bounded on sequences of points, Ann. Soc. Polon. Math. 22, 207-234.

J. Korevaar [1949b], A simple proof of a theorem of Pólya, Simon Stevin 26, 81-89.

E. Landau [1946], Darstellung und Begründung einiger neuerer Ergebnisse der Funktionentheorie, Chelsea, New York.

D. C. Lay [1970], Spectral analysis using ascent, descent, nullity and defect, Math. Ann. 184, 197-214.

G. K. Leaf [1963], A spectral theory for a class of linear operators, Pacific J. Math. 13, 141-155.

L. Leau [1899], Recherches sur les singularités d'une fonction définie par un développement de Taylor, J. Math. Pures Appl. 5, 365-425.

E. Le Roy [1900], Sur les séries divergentes et les fonctions définies par un développement de Taylor, Ann. Fac. Sci. Toulouse Math. 2, 317-430.

B. Ja. Levin [1964], Distribution of Zeros of Entire Functions, Amer. Math. Soc., Providence.

E. Lindelöf [1905], Le Calcul des Résidus, et ses Applications à la Théorie des Fonctions, Gauthier-Villars, Paris.

E. R. Lorch [1941], The integral representation of weakly almost-periodic transformations in reflexive vector spaces, Trans. Amer. Math. Soc. 49, 18-40.

C. Lubich and O. Nevanlinna [1991], On resolvent conditions and stability estimates, BIT 31, 293-313.

G. Lumer [1961], Semi-inner-product spaces, Trans. Amer. Math. Soc. 100, 29-43. 
G. Lumer [1964], Spectral operators, hermitian operators, and bounded groups, Acta Sci. Math. (Szeged) 25, 75-85.

G. Lumer [1971], Bounded groups and a theorem of Gelfand, Rev. Un. Mat. Argentina 25, 239-245.

G. Lumer and R. S. Phillips [1961], Dissipative operators in a Banach space, Pacific J. Math. 11, 679-698.

Yu. I. Lyubich and Vũ Quôc Phóng [1988], Asymptotic stability of linear differential equations in Banach spaces, Studia Math. 88, 37-42.

A. I. Markushevich [1976], Selected Chapters in the Theory of Analytic Functions, Nauka, Moscow (in Russian).

M. Mbekhta et J. Zemánek [1993], Sur le théorème ergodique uniforme et le spectre, C. R. Acad. Sci. Paris Sér. I Math. 317, 1155-1158.

C. A. McCarthy [1971], A strong resolvent condition does not imply power-boundedness, Chalmers Institute of Technology and the University of Göteborg, Preprint no. 15, Göteborg.

C. A. McCarthy and J. Schwartz [1965], On the norm of a finite Boolean algebra of projections, and applications to theorems of Kreiss and Morton, Comm. Pure Appl. Math. 18, 191-201.

P. Meyer-Nieberg [1991], Banach Lattices, Springer, Berlin.

A. Mokhtari [1988], Distance entre éléments d'un semi-groupe continu dans une algèbre de Banach, J. Operator Theory 20, 375-380.

V. Müller [1994], Local behaviour of operators, this volume, 251-258.

O. Nevanlinna [1993], Convergence of Iterations for Linear Equations, Birkhäuser, Basel.

T. Nieminen [1962], A condition for the self-adjointness of a linear operator, Ann. Acad. Sci. Fenn. Ser. A I No. 316, 5 pp.

J. I. Nieto [1982], Opérateurs à itérés uniformément bornés, Canad. Math. Bull. 25, 355-360.

N. K. Nikol'skiú [1977], A Tauberian theorem for the spectral radius, Siberian Math. J. 18, 969-972.

K. Noshiro [1960], Cluster Sets, Springer, Berlin.

N. Obreschkoff [1934], Lösung der Aufgabe 106, Jahresber. Deutsch. Math.-Verein. 43, 2. Abt., 13-15.

R. E. A. C. Paley and N. Wiener [1934], Fourier Transforms in the Complex Domain, Amer. Math. Soc., New York.

A. Pazy [1983], Semigroups of Linear Operators and Applications to Partial Differential Equations, Springer, New York.

D. Petz [1994], A survey of certain trace inequalities, this volume, 287-298.

G. Pólya [1931a], Aufgabe 105, Jahresber. Deutsch. Math.-Verein. 40, 2. Abt., 80.

G. Pólya [1931b], Aufgabe 106, ibid., 81.

G. Pólya [1974], Collected Papers, Vol. I, Singularities of Analytic Functions, R. P. Boas (ed.), The MIT Press, Cambridge, Mass.

G. Pólya und G. Szegö [1964], Aufgaben und Lehrsätze aus der Analysis, Springer, Berlin.

A. Pringsheim [1929], Kritisch-historische Bemerkungen zur Funktionentheorie, Sitzungsber. Bayer. Akad. Wiss. München, Math.-Natur. Abt., 95-124.

A. Pringsheim [1932], Vorlesungen über Funktionenlehre II.2, Teubner, Leipzig.

I. I. Privalov [1950], Boundary Properties of Analytic Functions, GITTL, Moscow (in Russian).

D. Przeworska-Rolewicz and S. Rolewicz [1987], The only continuous Volterra right inverses in $C_{c}[0,1]$ of the operator $\frac{d}{d t}$ are $\int_{a}^{t}$, Colloq. Math. 51, 281-285.

V. Pták [1976], The spectral radii of an operator and its modulus, Comment. Math. Univ. Carolin. 17, 273-279.

T. Pytlik [1987], Analytic semigroups in Banach algebras and a theorem of Hille, Colloq. Math. $51,287-294$

H. Radjavi and P. Rosenthal [1973], Invariant Subspaces, Springer, Berlin. 
M. Reed and B. Simon [1972], Methods of Modern Mathematical Physics, Vol. 1, Functional Analysis, Academic Press, New York.

R. Remmert [1991], Theory of Complex Functions, Springer, New York.

R. K. Ritt [1953], A condition that $\lim _{n \rightarrow \infty} n^{-1} T^{n}=0$, Proc. Amer. Math. Soc. 4, 898-899.

S. Rolewicz [1969], On orbits of elements, Studia Math. 32, 17-22.

H. C. Rönnefarth [1993], Charakterisierung des Verhaltens der Potenzen eines Elementes einer Banach-Algebra durch Spektraleigenschaften, Diplomarbeit, Technische Universität Berlin, Berlin, $77 \mathrm{pp}$.

G. Sansone [1959], Orthogonal Functions, Interscience, New York.

R. Sato [1979], The Hahn-Banach theorem implies Sine's mean ergodic theorem, Proc. Amer. Math. Soc. 77, 426 .

R. Sato [1981], On a mean ergodic theorem, ibid. 83, 563-564.

H. H. Schaefer [1974], Banach Lattices and Positive Operators, Springer, Berlin.

C. Schmoeger [1993], On isolated points of the spectrum of a bounded linear operator, Proc. Amer. Math. Soc. 117, 715-719.

S. L. Segal [1981], Nine Introductions in Complex Analysis, North-Holland, Amsterdam.

S. M. Shah [1946], On the singularities of a class of functions on the unit circle, Bull. Amer. Math. Soc. 52, 1053-1056.

S.-Y. Shaw [1980], Ergodic projections of continuous and discrete semigroups, Proc. Amer. Math. Soc. 78, 69-76.

A. L. Shields [1978], On Möbius bounded operators, Acta Sci. Math. (Szeged) 40, 371-374.

G. E. Shilov [1950], On a theorem of I. M. Gel'fand and its generalizations, Dokl. Akad. Nauk SSSR 72, 641-644 (in Russian).

V. S. Shul'man [1994], Invariant subspaces and spectral mapping theorems, this volume, 313325 .

A. M. Sinclair [1971], The norm of a Hermitian element in a Banach algebra, Proc. Amer. Math. Soc. 28, 446-450.

R. Sine [1969], A note on rays at the identity operator, ibid. 23, 546-547.

R. Sine [1970], A mean ergodic theorem, ibid. 24, 438-439.

B. M. Solom yak [1982], The existence of invariant subspaces for operators with nonsymmetric growth of the resolvent, Zap. Nauchn. Sem. Leningrad. Otdel. Mat. Inst. Steklov. (LOMI) 107, 204-208, 233-234 (in Russian).

B. M. Solomyak [1983], Calculuses, annihilators and hyperinvariant subspaces, J. Operator Theory 9, 341-370.

J. G. St a mpfli [1967], An extreme point theorem for inverses in a Banach algebra with identity, Proc. Cambridge Philos. Soc. 63, 993-994.

J. G. Stampfli and J. P. Williams [1968], Growth conditions and the numerical range in a Banach algebra, Tôhoku Math. J. 20, 417-424.

M. H. Stone [1948], On a theorem of Pólya, J. Indian Math. Soc. 12, 1-7.

J. C. Strikwerda [1989], Finite Difference Schemes and Partial Differential Equations, Wadsworth \& Brooks/Cole, Pacific Grove, Calif.

J. C. Strikwerd a and B. A. Wade [1991], Cesàro means and the Kreiss matrix theorem, Linear Algebra Appl. 145, 89-106.

A. Święch [1990], Spectral characterization of operators with precompact orbit, Studia Math. 96, 277-282; 97, 266

G. Szegö [1934], Lösung der Aufgabe 105, Jahresber. Deutsch. Math.-Verein. 43, 2. Abt., 10-11.

G. Szegö [1959], Orthogonal Polynomials, Amer. Math. Soc., New York.

B. Sz.-Nagy [1947], On uniformly bounded linear transformations in Hilbert space, Acta Sci. Math. (Szeged) 11, 152-157.

A. E. Taylor and D. C. Lay [1980], Introduction to Functional Analysis, Wiley, New York.

E. C. Titchmarsh [1939], The Theory of Functions, Oxford University Press. 
F. G. Tricomi [1955], Vorlesungen über Orthogonalreihen, Springer, Berlin.

L. Tschakaloff [1934], Zweite Lösung der Aufgabe 105, Jahresber. Deutsch. Math.-Verein. 43, 2. Abt., 11-13.

G. Valiron [1925], Sur la formule d'interpolation de Lagrange, Bull. Sci. Math. 49, 181-192, 203-224.

G. Valiron [1954], Fonctions Analytiques, Presses Universitaires de France, Paris.

J. A. Van Casteren [1985], Generators of Strongly Continuous Semigroups, Pitman, London.

I. Vidav [1956], Eine metrische Kennzeichnung der selbstadjungierten Operatoren, Math. Z. $66,121-128$.

I. Vidav [1982], Linear Operators in Banach Spaces, Društvo Matematikov, Fizikov in Astronomov SR Slovenije, Ljubljana (in Slovenian).

Vũ Quôc Phóng [1992], A short proof of the Y. Katznelson's and L. Tzafriri's theorem, Proc. Amer. Math. Soc. 115, 1023-1024.

Vũ Quôc Phóng [1993], Semigroups with nonquasianalytic growth, Studia Math. 104, 229241.

H.-D. Wacker [1985], Über die Verallgemeinerung eines Ergodensatzes von Dunford, Arch. Math. (Basel) 44, 539-546.

L. J. Wallen [1967], On the magnitude of $x^{n}-1$ in a normed algebra, Proc. Amer. Math. Soc. $18,956$.

J. Wermer [1952], The existence of invariant subspaces, Duke Math. J. 19, 615-622.

D. V. Widder [1941], The Laplace Transform, Princeton University Press, Princeton.

S. Wigert [1900], Sur les fonctions entières, Öfversigt af Kongl. Vetenskaps-Akademiens Förhandlingar, Stockholm, No. 8, 1001-1011.

W. Wils [1969], On semigroups near the identity, Proc. Amer. Math. Soc. 21, 762-763.

F. Wolf [1957], Operators in Banach space which admit a generalized spectral decomposition, Indag. Math. 19, 302-311.

N. J. Young [1978], Analytic programmes in matrix algebras, Proc. London Math. Soc. 36, $226-242$.

B. Zalar [1993], History of the Vidav theorem, Obzornik Mat. Fiz. 40, 9-14 (in Slovenian).

M. Zarrabi [1993], Contractions à spectre dénombrable et propriétés d'unicité des fermés dénombrables du cercle, Ann. Inst. Fourier (Grenoble) 43, 251-263.

M. Zarrabi [to appear], Spectral synthesis and applications to $C_{0}$-groups, J. Austral. Math. Soc. Ser. A.

J. Zemánek [à paraître], Sur les itérations des opérateurs, Publ. Math. Univ. Pierre et Marie Curie, Séminaire d'Initiation à l'Analyse.

X.-D. Zhang [1992], Two simple proofs of a theorem of Schaefer, Wolff and Arendt, Indag. Math. 3, 247-250 